Case Report:

\title{
Torticollis as a Rare Presentation of Cerebellopontine Angle Choroid Plexus Papilloma in Children
}

\author{
Mohammad Faraji-Rad $^{1}$, Einoallah Alipour ${ }^{2^{*}}$, Elnaz Faraji-Rad ${ }^{3}$ \\ 1- Professor of Neurosurgery, Fellowship in Skull Base Surgery and Neurovascular Surgery, Department of Neurosurgery, Qaem Hospital, Mashhad \\ University of Medical Sciences, Mashhad, Iran \\ 2- Resident of Neurosurgery, Department of Neurosurgery, Qaem Hospital, Mashhad University of Medical Sciences, Mashhad, Iran , \\ 3- Nuclear Medicine Resident, Department of Medical Imaging, LHSC Victoria Hospital, Western University, London, Ontario, Canada
}

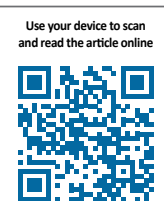

Citation: Faraji-Rad M, Alipour E, Faraji-Rad E. Torticollis as a Rare Presentation of Cerebellopontine Angle Choroid Plexus Papilloma in Children. Iran J Neurosurg. 2020; 6(4):219-224. http://dx.doi.org/10.32598/irjns.6.4.6

http://dx.doi.org/10.32598/irjns.6.4.6

Article info:

Received: 19 May 2020

Accepted: 16 Jul 2020

Available Online: 01 Oct 2020

Keywords:

Choroid plexus papilloma, Torticollis, Cerebellopontine angle, Brain stem

\section{ABSTRACT}

Background and Importance: The rare intracranial neoplasms are Choroid Plexus Papillomas (CPPs), especially in the cerebellopontine angle. The main location of choroid plexus papillomas in adults and children are $4^{\text {th }}$ ventricle and lateral ventricles, respectively.

Case Presentation: We report on a little girl with a cerebellopontine angle CPP who presented with symptoms of torticollis. Assessment of Magnetic Resonance Imaging showed a mass in the right cerebellopontine angle, next to the brain stem. The tumor was completely resected using the right retrosigmoid approach method. A pathological examination determine a typical CPP that this being should be considered an extremely rare cause of a lesion in the posterior fossa.

Conclusion: CPP is usually presented in the atrium of the lateral ventricle in children; however, we reported a rare case in the cerebellopontine angle. 


\section{Highlights}

- CPP usually affects in the atrium of the lateral ventricle in children.

- Rarely, CPP may present in the cerebellopontine angle.

- The prognosis of CPP is good, with a $90 \%$ survival rate.

\section{Plain Language Summary}

A type of tumor named Choroid Plexus Papillomas (CPPs) originates from the lining of tissue in the brain. In fact, these tumors are usually located in the $4^{\text {th }}$ ventricle and in the lateral ventricles in adults and children. However, we reported a rare case in a 2-year-old boy that was presented with the cerebellopontine angle of the brain. This case report is important to provide a better understanding of the tumor presentation in this part and the subsequent management.

\section{Background and Importance}

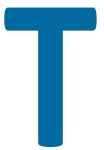

he rare intraventricular tumors are choroid plexus tumors that comprise less than $1 \%$ of all intracranial tumors and $2 \%-4 \%$ of brain tumors in children [1, 2]. They are most commonly located in the lateral ventricles of children and infants and the $4^{\text {th }}$ ventricle in adults [2, 3]. The cerebellopontine angle is a rare location for the CPP and only a limited number of Choroid Plexus Papillomas (CPPs) have been reported at this location [4-6]. The surgery of this tumor is challenging as patients are typically young, overproduction of Cerebrospinal Fluid (CSF) after surgery, and extreme vascularity of the tumor, which can lead to significant blood loss during surgery and incomplete resection of these tumors [7-9]. Here, we reported on a case of cerebellopontine angle choroid plexus papilloma which was resected by the retrosigmoid approach.

\section{Case Presentation}

We hospitalized a 2-year-old female patient who presented with torticollis for 15 days. On physical examination, the child was conscious, oriented, and without any neurological deficit. Magnetic Resonance Imaging (MRI) demonstrated a cystic mass without enhancement and restriction in the right posterior fossa next to the brain stem with pressure effect on the fourth ventricle (Figure 1). Based on the radiographic appearances of the lesion, a definite diagnosis of choroid plexus papilloma was reported.

At surgery, a retrosigmoid craniotomy was performed and the tumor was approached through a retrosigmoid approach. A cystic mass was observed in the right cer- ebellopontine angle next to the brain stem. The tumor was cystic and the gross total resection of the tumor was performed. Fortunately, the patient tolerated the procedure well during the operation. Post-operatively, neurological deficits were observed in the patient. Histopathology was consistent with the diagnosis of choroid plexus papillomas (Figure 2 ).

\section{Discussion}

Choroid plexus papilloma is one of the common neoplasms. It occurrence mainly within the ventricular system and involvement of the cerebellopontine angle is rare. During childhood, $80 \%$ of CPPs are found in the lateral ventricles, $16 \%$ in the $4^{\text {th }}$ ventricle, and $4 \%$ in the 3rd ventricle. These tumors are generally more common in males than females $[3,10,11]$.

Choroid plexus papillomas are generally classified as benign neoplasms, defined by World Health Organization (WHO) as Grade I and Grade II. Resection of these tumors usually cures the disease and recurrence after gross total resection is rare. As these tumors are highly vascular, they involve young people and may cause post-operative complications such as CSF overproduction, their management is usually challenging $[2,7,12]$. These lesions are highly vascular with several anastomosis of choroidal blood vessels and may affect interventricular space. Thus, adjuvant endovascular embolization can reduce blood loss [9].

Several metastases of Cerebellopontine Angle (CPA) is also probable in this region that are mainly from the neuro-ectodermal origin. Cancers that are reported in the literature to have metastasis to the CPA include 


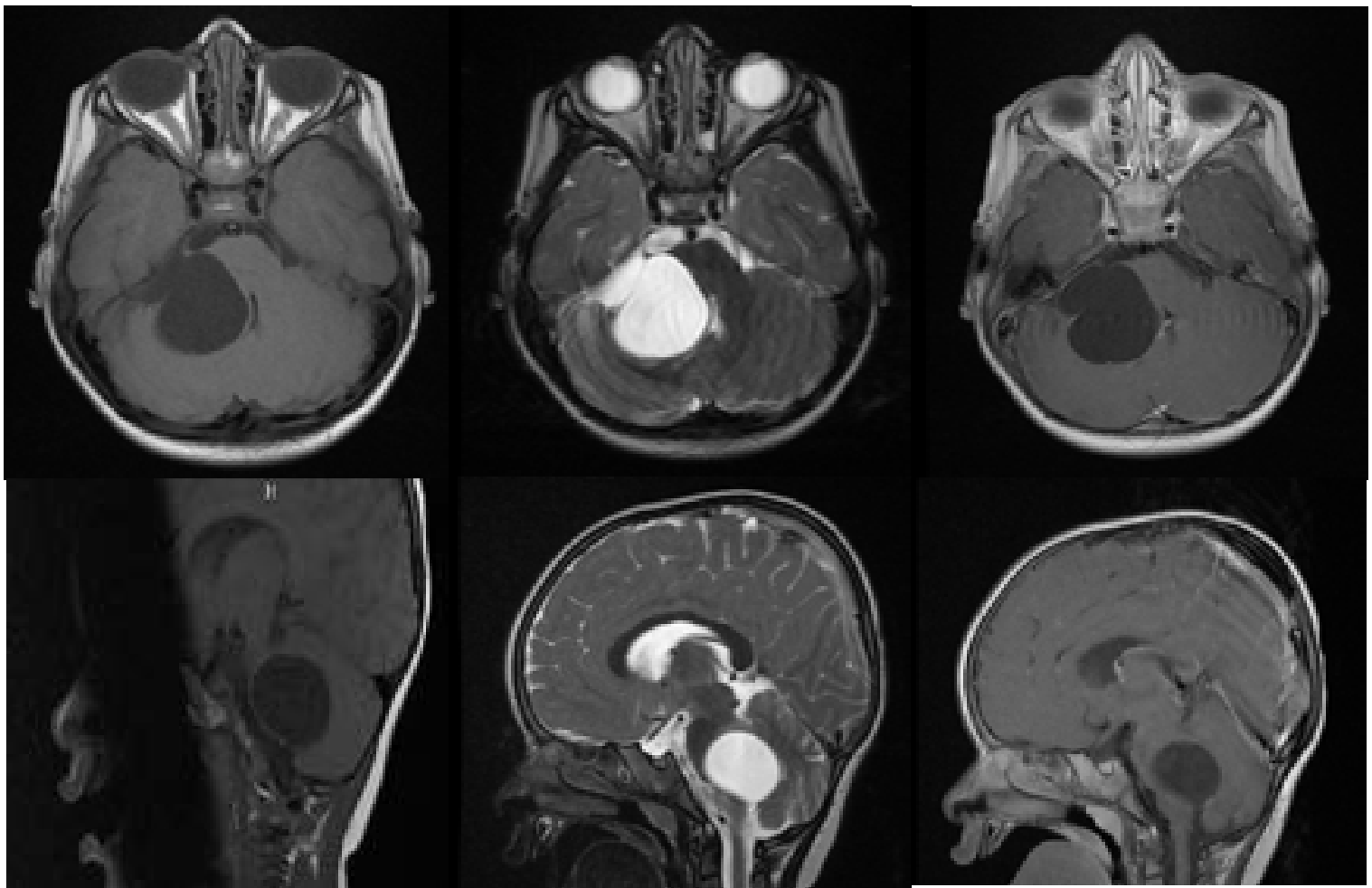

NS

Figure 1. Pre-operative T1, T2, and Gad-MRI showing the tumor without enhancement in the right posterior fossa next to the brain stem with pressure effect on the $4^{\text {th }}$ ventricle

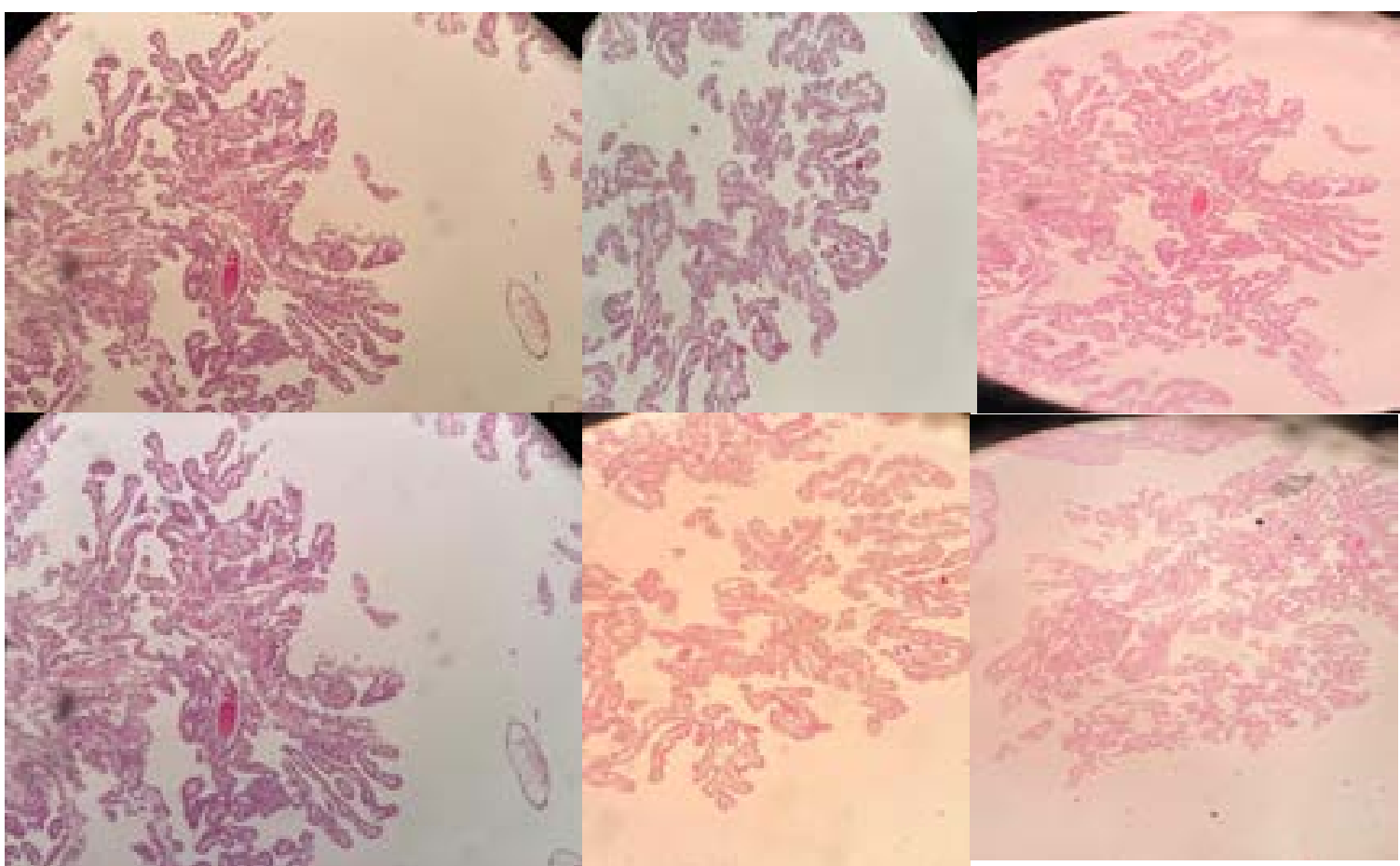

Figure 2. Choroid plexus papilloma showing a single layer of orderly cuboidal to columnar epithelial cells stays on distinct fibrovascular cores. Inset shows a hyalinized blood vessel 
prostate, breast, lung, ocular melanoma, and nasopharynx cancer. Moreover, cases of hematologic cancer with metastasis to CPA also are reported. Therefore, these should be considered in the differential diagnosis of a mass in this region [1].

The treatment of CPP is the surgical excision for the resection of these tumors. The complete tumor should be removed and is usually accessible in as many as $96 \%$ of cases. Most of the challenges that neurosurgeons face regarding the management of this type of tumor are related to the heavy blood loss in children, who have a proportionally small circulating blood volume. Although mortalities due to all causes comprise between 0 to $25 \%$, up to $12 \%$ of mortalities are reported to be caused by blood loss $[2,7,12,13]$.

Various surgical approaches have been described for cerebellopontine angle tumors. We usually prefer a retrosigmoid approach to a CP angle tumor. The effect of gravity in sitting position and deep venous structures which lie dorsal to the tumor are advantages of using this approach. Yet, the narrow operative corridor which can cause problems in resecting large vascular tumors is disadvantageous. Prevention of bleeding is a major consideration during surgical resection of these lesions in pediatric patients, thus a technique of gentle, repetitive bipolar coagulation of the tumor surface under permanent irrigation (to shrink the tumor and remove it totally) is preferred to its piecemeal resection. Gross total resection of the tumor brings a long-term survival of 90 to $100 \%$. Those malignant tumors or those with extension to leptomeningeal need adjuvant therapy $[7,12]$.

\section{Conclusion}

We presented a 2-year-old girl with CPP who presented with symptoms of torticollis. The imaging determined a mass in the right cerebellopontine angle next to the brain stem. We often surgically cure choroid plexus papillomas. The most common location of CPP is in the atrium of the lateral ventricle in children. CPPs have a nearly $90 \%$ survival after tumor resection. Although, we reported a rare case of CPP in the cerebellopontine angle.

\section{Ethical Considerations}

Compliance with ethical guidelines

The participants were informed of the purpose of the research and its implementation stages. A written consent has been obtained from the subjects. They were also assured about the confidentiality of their information and were free to leave the study whenever they wished, and if desired, the research results would be available to them.

Funding

This research did not receive any grant from funding agencies in the public, commercial, or non-profit sectors.

\section{Authors contributions}

Conception and design: Mohammad Faraji-Rad; Data collection: Mohammad Faraji-Rad; Analysis and interpretation: Einoallah Alipour; Drafting the article: Mohammad FarajiRad; Critically revising the article: Einoallah Alipour; Reviewing submitted version of manuscript: Einoallah Alipour; Approving the final version of the manuscript: Elnaz Farajirad.

\section{Conflict of interest}

The authors have no conflicts of interest in the present study.

\section{References}

[1] Buckle C, Smith JK. Choroid plexus papilloma of the third ventricle. Pediatric Radiology. 2007; 37(7):725. [DOI:10.1007/ s00247-007-0474-5] [PMID]

[2] Ogiwara H, Dipatri AJ Jr, Alden TD, Bowman RM, Tomita T. Choroid plexus tumors in pediatric patients. British Journal Neurosurgery. 2012; 26:32-7. [DOI:10.3109/02688697.2011.601 820] [PMID]

[3] Kroppenstedt SN, Golfinos J, Sonntag VK, Spetzler RF. Pineal region lesion masquerading choroid plexus papilloma: Case report. Surgical Neurology. 2003; 59(2):124-7. [DOI:10.1016/ S0090-3019(02)00988-6]

[4] Nakano I, Kondo A, Iwasaki K. Choroid plexus papilloma in the posterior third ventricle: Case report. Neurosurgery. 1997; 40(6):1279-82. [DOI:10.1097/00006123-199706000-00030] [PMID]

[5] Pawar SJ, Sharma RR, Mahapatra AK, Lad SD, Musa MM. Choroid plexus papilloma of the posterior third ventricle during infancy and childhood: Report of two cases with management morbidities. Neurology India. 2003; 51(3):379-82. [PMID]

[6] Gupta P, Sodhi KS, Mohindra S, Saxena AK, Das A, Khandelwal N. Choroid plexus papilloma of the third ventricle: A rare infantile brain tumor. Journal of Pediatric Neurosciences. 2013; 8(3):247-9. [DOI:10.4103/1817-1745.123696] [PMID] [PMCID]

[7] Krishnan S, Brown PD, Scheithauer BW, Ebersold MJ, Hammack JE, Buckner JC. Choroid plexus papillomas: A single institutional experience. Journal of Neuro-Oncology. 2004 68(1):49-55. [DOI:10.1023/B:NEON.0000024745.06073.07] [PMID] 
[8] Tsumoto T, Nakai E, Uematsu Y, Nakai K, Itakura T. [Choroid plexus papilloma in the posterior third ventricle in infancy: A case report (Japanese)]. No Shinkei Geka. Neurological surgery. 1999; 27(7):673-8. [PMID]

[9] Wind JJ, Bell RS, Bank WO, Myseros JS. Treatment of third ventricular choroid plexus papilloma in an infant with embolization alone. Journal of Neurosurgery Pediatrics. 2010; 6(6):579-82. [DOI:10.3171/2010.9.PEDS1039] [PMID]

[10] Carson BS, Weingart JD, Guarnieri M, Fisher PG. Third ventricular choroid plexus papilloma with psychosis. Case report. Journal of Neurosurgery. 1997; 87(1):103-5. [DOI:10.3171/jns.1997.87.1.0103] [PMID]

[11] Karim A, Fowler M, McLaren B, Cardenas R, Patwardhan $R$, Nanda A. Concomitant choroid plexus papillomas involving the third and fourth ventricles: A case report and review of the literature. Clinical Neurology and Neurosurgery. 2006; 108(6):586-9. [DOI:10.1016/j.clineuro.2005.04.008] [PMID]

[12] Menon G, Nair SN, Baldawa SS, Rao RB, Krishnakumar KP, Gopalakrishnan CV. Choroid plexus tumors: An institutional series of 25 patients. Neurology India. 2010; 58(3):429-35. [DOI:10.4103/0028-3886.66455] [PMID]

[13] Sarkar C, Sharma MC, Gaikwad S, Sharma C, Singh VP. Choroid plexus papilloma: A clinicopathological study of 23 cases. Surgical Neurology. 1999; 52(1):37-9. [DOI:10.1016/ S0090-3019(99)00049-X] 
This Page Intentionally Left Blank 